\title{
Shape constancy: The effects of changing shape orientation and the effects of changing the position of focal features
}

\author{
GLYN W. HUMPHREYS \\ Birkbeck College, University of London, London, England
}

\begin{abstract}
Five experiments examined the time taken to judge that two consecutive elongated geometrical shapes had the same structure, irrespective of their orientation. Shape transformations either changed the orientation of the principal axis while maintaining the relative locations of focal features or maintained the orientation of the principal axis while changing the relative locations of focal features, or they changed both. Experiment 1 demonstrated that changes in the orientation of the principal axis were more detrimental to matching than were changes in the locations of the shape's focal features. Indeed, the time taken to match same-orientation shapes was the same as that taken to match shapes that maintained the same position in the visual field. Further experiments showed that this result was not due to differential apparent motion in the transformation conditions, that it was not due to response bias, and that it generalized across shapes. However, the result was different when subjects could predict the location of the to-be-matched stimulus. In this case, performance was principally affected by the position of the focal feature of the shape and not by the shape's orientation. It is suggested that the results reflect the efficiency with which subjects can construct matching representations for the stimuli. When subjects cannot predict stimulus locations, they generate representations by describing shape structure relative to the shape's principal axis. When the axis of the to-be-matched shapes is constant, subjects can use the same procedure in generating this representation for both shapes, facilitating matching relative to the case in which the orientation of the axis changes. When subjects can predict the stimulus location, they selectively attend to the focal features of shapes, minimizing the effects of shape orientation.
\end{abstract}

People can recognize familiar shapes when they are seen from novel viewpoints. This ability to achieve shape constancy even when the retinal projection changes is a primary characteristic required of any visual processing system, and should be accounted for by any theory of shape recognition (Marr, 1980). The present paper explores some of the processes that enable shape constancy to be achieved.

Studies of the efficiency with which subjects achieve shape constancy across a range of stimulus transformations can inform us about the nature of the processes involved. A common task in such studies is simultaneous or successive form matching, in which subjects decide whether two forms have the same identity. A number of studies have investigated the effects, on matching, of transformations in size (e.g., Bundesen \& Larsen, 1975; Larsen \& Bundesen, 1978; Sekuler \& Nash, 1972) or orientation (e.g., Cooper

This work was supported by a grant from the Social Science Research Council. I would like to thank Penny Wilcox for her help in producing the presentation programs, Philip Quinlan and Jane Riddoch for their discussion of earlier drafts of this paper, and an anonymous referee for helpful comments during its processing. My mailing address is: Department of Psychology, Birkbeck College, University of London, Malet Street, London, WC1E 7HX, England.
\& Shepard, 1973, 1975; Metzler \& Shepard, 1974; Shepard, 1975; Shepard \& Metzler, 1971). In some tasks, subjects discriminate between transformed shapes and their reflections, knowing the structural identity of the shapes and the type of transformation by which they will differ. For instance, Shepard and Metzler (1971) presented subjects with perspective line drawings of three-dimensional shapes at different orientations and asked them to decide whether the shapes were physically the same or whether one was a reflection of the other. A common finding is that reaction time (RT) to match forms is a linear function of the size of the transformation, suggesting that shape constancy is achieved by the transformation of an internal representation of a shape in a manner isomorphic with the external transformation.

However, this is not the only, or perhaps even the usual, means of achieving shape constancy. There are cases in which subjects cannot predict the transformation or the internal representation appropriate to a shape. Shape constancy in this circumstance requires the generation of a representation that is viewpoint-independent from one that is viewpointdependent. For instance, Rock, DiVita, and Barbeito (1981) showed subjects novel two- or three-dimensional wire figures, and then tested for the recognition of 
the figures following an orientation transformation. They found that altering the relative positions of the top and bottom of the object was more detrimental to recognition than altering only the positions of the sides. This result led Rock et al. to argue that shapes were represented by a description of their top and bottom features and the location of the sides between the top and bottom. Consequently, the most disruptive orientational transformation will alter the relative positions of the top and bottom features.

A related proposal concerning the processing of simple geometric shapes has been made by Braine (1978; Braine, Relyea, \& Davidman, 1981). She, like Rock et al., argues that shapes are recognized by a hierarchical process of feature description; however, she proposes that this process first codes only the top position, and then proceeds in a top-to-bottom direction. The top position is characterized by the presence of a focal feature and by the vertical orientation of the main axis of the shape. Because the feature-description process is hierarchical, recognition will proceed most efficiently when the focal feature is at the top of the shape. Transformations producing a change in the relative position of the top of the shape will most affect recognition.

The proposals of Rock et al. and Braine maintain that shapes are represented internally by a structural description based on important characteristics of the shape. They differ in the importance assigned to bottom-position features and in specifying a means by which the top position can be assigned. However, both suggest that the top-position feature provides the primary reference coordinates for the representation. Because the coordinate system is then based on a feature of the object, the representation so formed is viewpoint-independent and so would enable shape constancy to occur. Transformations generating changes in viewpoint-dependent codings of the (top) reference feature will give rise to problems in deriving shape constancy.

Work in the field of computer vision also suggests that shape constancy depends on a structural description based on an object-centered coordinate system (e.g., Hinton, 1981; Marr, 1979, 1980, 1982; Marr \& Nishihara, 1978). However, this approach contrasts with the feature-based account in proposing that the coordinate system is derived from a more global property of the shape than the position of focal features. For instance, Marr and his co-workers have argued that a suitable coordinate system may be determined by the orientation of the principal axis of the shape (Marr, 1982; Marr \& Nishihara, 1978). According to this account, changes in the orientation of a shape may be more disruptive to shape constancy than changes in the position of focal features.

It is difficult to apply this argument directly to Rock et al. experiment because the effects of the transformations on the principal axis were not directly controlled. Nevertheless, many of the transformations producing changes of the top and bottom positions also appear to have changed the orientation of the principal axis (see Figures 1 and 2 in Rock et al., 1981). It seems reasonable to conclude that their findings are consistent with these proposals.

There are also other examples supporting the axisbased account. For instance, consider the shapes presented in Figure 1. Mach (1897) first noted that, despite the fact that all the individual shapes were at the same orientation, subjects reported that the isolated shape was seen as a diamond whereas the shapes in the oblique line were seen as squares. The different descriptions for these shapes appear to arise because the shapes in the oblique line are represented relative to a coordinate system based on the elongated axis of the configuration; in contrast, the isolated shape is represented relative to its salient vertical axis of symmetry (cf. Goldmeier, 1936; Julesz, 1971; Rock \& Leaman, 1963). Relative to these axes, descriptions appropriate to a square and a diamond arise. Consistent empirical evidence has been reported by Humphreys (1983). Subjects had to decide as quickly as possible whether successive forms had the same or different numbers of sides. Two types of shape were used, those with an unambiguous, elongated principal axis (isosceles triangles and elongated pentagons) and those with an ambiguous principal axis (squares and regular hexagons). Matching shapes could be orientational transformations of one another, so the task demanded matches of shape identity independent of the transformation. Humphreys found that orientational transformations that changed the axis aligned with the vertical disrupted the matching of shapes with ambiguous principal axes (e.g., when a square was transformed $45 \mathrm{deg}$ within-the-plane, into a diamond). Similar transformations did not disrupt the matching of shapes with an unambiguous principal axis (e.g., when an isosceles triangle was transformed $45 \mathrm{deg}$ within-the-plane). These data suggest that shapes were matched using a coordinate system derived from the shape's principal axis. For shapes with an ambiguous principal axis, the axis of description will be determined by alignment of the

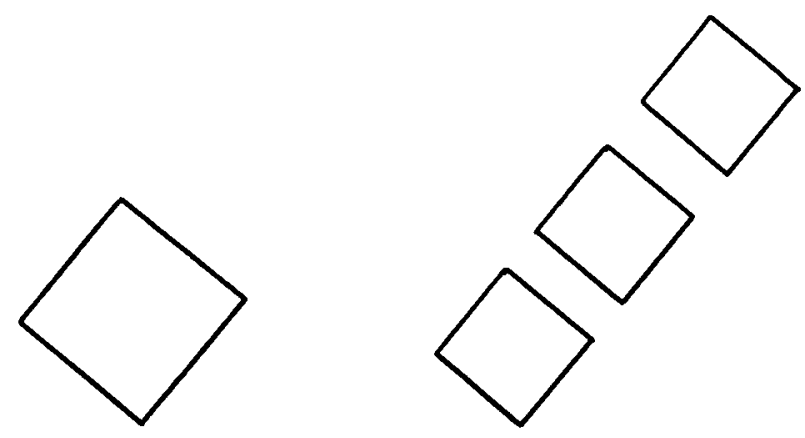

Figure 1. Mach's demonstration. 
axis with the vertical. Changing the shape description relative to this axis disrupts matching.

Unfortunately, it is likely that the manipulations used by Humphreys (1983) not only altered the orientation of the principal axis of the stimuli but also the position of their focal features. For instance, the shapes with ambiguous principal axes probably had ambiguous focal features (e.g., top horizontal line vs. top apex for normal and transformed squares), whereas this was not so for the shapes with an unambiguous principal axis (e.g., an isosceles triangle). If the orientational transform that aligned different axes with the vertical also aligned different focal features, it is possible that the disruption to matching was due to a change in the focal feature.

The present paper set out to test whether transformations that change the relative orientation of a shape's principal axis affect shape constancy more than transformations that change the relative locations of its focal feature. The aim was to evaluate which of these two properties is used to derive the coordinate system for viewpoint-independent shape representation.

\section{EXPERIMENT 1}

In order to test whether a change in the orientation of a shape's principal axis or a change in the position of its focal features is the more disruptive to shape constancy, Experiment 1 pitted the two factors against each other. A successive form-matching task, similar to that used by Humphreys (1983), was employed. Either single triangles or single parallelograms were presented, and subjects decided whether successive shapes had the same or a different number of sides. Matching shapes were either identical or orientational transforms of one another. Examples of the shapes and the transformations are shown in Figure 2.

The transformations were chosen to vary either the orientation of the shape or the position of its focal feature. The predictions of the axis-based and the feature-based theories differ over which of these manipulations will most af fect shape matching.

For the triangles, the feature-based account predicts that transformation $a$ should be less disruptive than transformations $b$ and $c$, because the position of the focal feature in the triangle remains at the top or the bottom following only transformation $a$. Transformation $b$ may also be less disruptive than transformation $c$, because the position of the focal feature remains on either the right or left in the former but not the latter condition. In contrast, the axis-based account proposes that transformation $c$ should be least disruptive because only it maintains the orientation of the principal axis.

Parallelograms, unlike triangles, do not contain a focal feature, and whichever features fall at the top of the shape may be used as the primary reference coordinates. Transformations $a$ and $b$ alter the left/
A. Parallelogram transformations

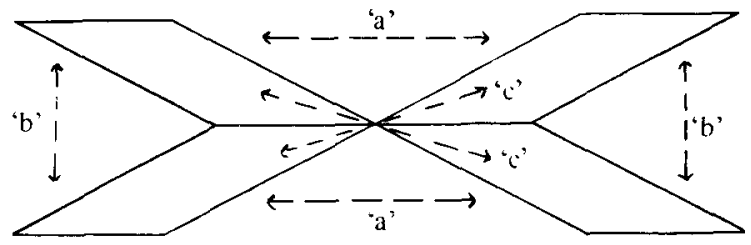

B. Triangle transformations

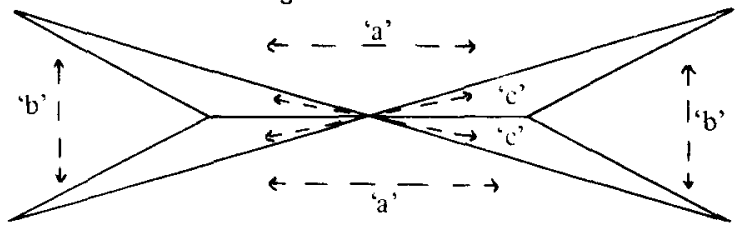

Figure 2. The shapes and transformation conditions used in Experiment 1.

right coding of the angles at the top of the shape and therefore may produce some change in the local feature descriptions; they also alter the orientation of the shape's principal axis. Transformation $c$ alters only the absolute position of the shape in the visual field. Both the axis-based account and the featurebased account predict that transformations $a$ and $b$ will be more disruptive to matching than transformation $c$.

\section{Method}

Unless otherwise stated, the same method was used in Experiments 2-5.

\section{Subjects}

There were 16 paid members of the Birkbeck College subject pool, 10 females and 6 males. Each subject took part in one experimental session lasting about $\mathbf{4 0} \mathrm{min}$. All subjects had normal or corrected-to-normal vision.

\section{Apparatus and Stimuli}

Stimulus presentation, timing, and data collection were controlled by a PDP-11/10 computer. Stimuli were plotted on an Advanced Instruments oscilloscope (02 250) equipped with a P-31 rapid-decay phosphor. The subjects viewed the display from a distance of $50 \mathrm{~cm}$, and their heads were fixed in a vertical position by means of a cushioned headrest; they fixated a central cross subtending a visual angle of $0.5 \mathrm{deg}$ horizontally and vertically. The shapes were plotted as follows: The first shape was plotted in the right upper quadrant of the screen relative to the central cross, with the bottom left point of the shape falling on the same coordinate as the center of the cross. This shape was successively rotated to $180 \mathrm{deg}$ about the $\mathrm{Y}$-axis, to $180 \mathrm{deg}$ about the $\mathrm{X}$-axis, and to $180 \mathrm{deg}$ about the $\mathrm{Y}$-axis and $180 \mathrm{deg}$ about the $\mathrm{X}$-axis, to create the reflections in left upper, right lower, and left lower quadrants, respectively. This procedure was conducted for both the parallelograms and the triangles.

The parallelograms had 2-cm-long bases and 3-cm-long sides, and the bottom left acute angle of the first plotted shape (right upper quadrant) was $30 \mathrm{deg}$. The right upper quadrant triangle had the same base length as the equivalent parallelogram $(2 \mathrm{~cm})$, and the top apex of the triangle fell at the top right-hand corner of the parallelogram. The bottom left acute angle of the first 
plotted triangle was thus $15 \mathrm{deg}$. The parallelogram and triangle stimuli so created have salient elongated axes of symmetry.

The subjects made manual buttonpress responses, and a given response caused an interrupt in the presentation program enabling RT to be measured from the onset of the second (target) stimulus.

\section{Design}

Each stimulus was paired with every other stimulus equally often, creating four conditions in which consecutive shapes (ST1 and ST2) had the same number of sides. These conditions, shown in Figure 3, were: (1) same position (ST1 and ST2 identical), (2) vertical-axis transformation ( $a, \mathrm{ST} 1$ in the right upper or lower quadrant and ST2 in the left upper or lower quadrant, or vice versa), (3) horizontal-axis transformation $(b, \mathrm{ST} 1$ in the right or left upper quadrant and ST2 in the right or left lower quadrant, or vice versa), and (4) same orientation (c, ST1 in the right or left upper quadrant and ST2 in the left or right lower quadrant, or vice versa). The same four transformation conditions occurred when consecutive shapes had different numbers of sides.

\section{Procedure}

The subjects were instructed to match consecutive shapes on the basis of whether they both contained three or both contained four sides ("same" response) or whether one had three and the other four sides ("different"' response). They were told the set of shapes and asked to make the same/different decision as quickly as possible without making errors. Half the subjects responded "same" with the preferred hand and "different" with the nonpreferred hand; the hand-response assignment was reversed for the other subjects.

Immediately following a response, the subjects were presented with feedback about their response accuracy. Feedback was the word CORRECT or ERROR, which appeared on the scope during the intertrial interval of $1.5 \mathrm{sec}$.

There were 512 experimental trials presented in four separate blocks (128 trials per block). Within each block, each stimulus was paired with each other stimulus and occurred first or second in this pairing twice (16 trials per condition per block). The subjects initiated a block of trials by pressing both response buttons simultaneously; once initiated, though, display timing throughout the block was controlled by the computer. On each trial, the fixation cross was presented for $500 \mathrm{msec}$, followed by ST1 for $50 \mathrm{msec}$, an ISI of $200 \mathrm{msec}$, during which the screen was blank, and ST2 for $200 \mathrm{msec}$. These display timings have been shown to produce reliable transformation effects on successive form-matching performance (Humphreys, 1983). The order of presentation of the shape pairs within each block was randomized for each subject by computer.

Prior to the experimental trials, the subjects were given 48 practice trials, in which each condition was presented six times.

\section{Results}

The mean RTs in each condition were calculated for each subject. RTs \pm 3 SD from each subject's mean in each condition were discarded along with all error responses. Since "same" and "different" responses may depend on different underlying processes (see, e.g., Bamber, 1969), these responses were analyzed separately. This procedure was followed in all the present experiments.

\section{RT Data: “Same”' Responses}

Mean correct RTs and percentage errors for "same" responses are shown in Table 1.

The data were submitted to a two-way repeated measures analysis of variance, with shape (parallelograms and triangles) and condition (same position, vertical-axis transformation, horizontal-axis transformation, and same orientation) as fixed effects and subjects as a random factor. There was a statistically significant main effect of conditions $[F(3,45)=13.32$, $\mathrm{p}<.001]$. Neither the main effect of shape nor the conditions $\times$ shape interaction was reliable $[F(1,15)$ $=1.09, \mathrm{p}>.05$, and $\mathrm{F}<1.0$, respectively].

The conditions effect was further investigated using the Newman-Keuls technique. RTs in the same-position and the same-orientation conditions were faster than those in the vertical-axis and the horizontal-axis transformation conditions (all comparisons $p<.01$ ). However, there was no difference between the sameposition and the same-orientation conditions, or between the vertical-axis and the horizontal-axis conditions.

\section{RT Data: “Different"' Responses}

"Different" responses were separated according to whether ST2 was a parallelogram (and ST1 a triangle) or whether ST2 was a triangle (and ST1 a parallelogram). Mean correct RTs and percentage errors as a function of ST2 shape and the transformation condition are shown in Table 1.

Table 1

Mean Correct RTs (in Milliseconds) and Percentage Error (PE) on "Same" Response Trials, as a Function of Shape and Transformation Condition, and on "Different" Response Trials, as a Function of ST2 Shape and Transformation Condition, in Experiment 1

\begin{tabular}{|c|c|c|c|c|}
\hline & \multicolumn{2}{|c|}{ Parallelogram } & \multicolumn{2}{|c|}{ Triangle } \\
\hline & RT & $\mathrm{PE}$ & RT & PE \\
\hline \multicolumn{5}{|c|}{ "Same" Response Trials } \\
\hline Same Position & 425 & 0.6 & 412 & 0.7 \\
\hline Vertical-Axis Transformation & 461 & 2.1 & 440 & 1.8 \\
\hline Horizontal-Axis Transformation & 463 & 2.3 & 453 & 2.2 \\
\hline Same Orientation & 421 & 1.2 & 418 & 1.3 \\
\hline \multicolumn{5}{|c|}{ "Different" Response Trials } \\
\hline Same Position & 487 & 1.6 & 484 & 1.8 \\
\hline Vertical-Axis Transformation & 475 & 1.8 & 471 & 1.3 \\
\hline Horizontal-Axis Transformation & 476 & 1.5 & 462 & 1.5 \\
\hline Same Orientation & 484 & 1.8 & 472 & 1.9 \\
\hline
\end{tabular}


A two-way repeated measures analysis of variance was conducted, with ST2 shape and transformation condition as fixed effects and subjects as a random effect. The main effects of shape and condition did not approach significance [F $<1.0$ and $F(3,45)=$ $1.24, \mathrm{p}>.05$, respectively]. The shape $\times$ condition interaction was similarly nonsignificant $(F<1.0)$.

\section{Error data}

Relatively low error rates were recorded. In all cases, the error data followed the same pattern as the RT data (see Table 1). Because of this, no analysis of the error data was carried out. RTs were not traded off with response accuracy.

\section{Discussion}

Transformations of triangles that maintain the orientation of the principal axis while changing the position of focal features from the top or bottom of the shape (in the same-orientation condition) disrupt shape matching less than transformations that change the orientation of the principal axis while maintaining the position of the focal feature at the top or bottom of the shape (in the vertical-axis condition). The transformation in the same-orientation condition also disrupted shape matching less than the transformation in the horizontal-axis condition, which changed the orientation of the shape but maintained the focal feature at the left or right of the shape. This finding suggests that the orientation of the principal axis plays a primary role in shape matching.

Interestingly, RTs to match shapes in the sameorientation condition were not different from RTs to match identical shapes. Shape matching was not facilitated by having shapes in the same position in the visual field. Also, the transformation effects were equivalent for parallelograms and for triangles, even though the triangles changed their top-bottom direction in the same-orientation condition whereas the parallelograms did not. Table 1 shows that the direction change apparently had a small detrimental effect on RTs to match triangles (indicated by the differences between the same-orientation and the sameposition conditions and between the horizontal-axis and the vertical-axis conditions). However, this effect was not reliable.

The data indicate that shape matching could not be made to operate here by subjects transforming their internal representation of ST1 isometrically to the external transformation of ST1 into ST2 (cf. Shepard \& Metzler, 1971). If this were the case, RTs in the same-orientation condition should not have been equivalent to RTs when ST1 and ST2 were in the same position. Indeed, RTs should have been slowest in the same-orientation condition because that involved a larger rotation than those present in the other conditions (180 deg about the vertical plus $180 \mathrm{deg}$ about the horizontal).

Other accounts of how shape orientation affects matching differ in their predictions about the effects of the transformations on "different" response RTs. One possibility is that shapes in the same orientation appear more similar. It is well established that the visual system responds rapidly to orientation information and that orientation differences are strong segmentation cues (Beck, 1972; Julesz, Gilbert, Shepp, \& Frisch, 1973). It could be that constancy of orientation here biases a "same" response and that orientation change biases a "different" response. If "samedifferent" decisions are made when sufficient evidence is accumulated to exceed a criterion set for either response (Krueger, 1978), "same" responses will be faster in the same-orientation condition than when the orientation changes. This account predicts that, on "different" trials, RTs should be slower when the shapes are in the same orientation relative to the different orientation conditions.

A second possibility is that presenting any two shapes in the same orientation in some way facilitates their processing. For instance, it could be that subjects generate the description of a shape relative to its principal axis and that information about the orientation of the axis is maintained along with the shape description. In this case, the processing of two shapes in the same orientation may be facilitated because subjects do not have to recompute orientation information. This account predicts that "different" RTs to shapes in the same orientation should be faster than "different" RTs to shapes in different orientations (i.e., in the vertical-axis and horizontal-axis conditions).

Both of the above accounts depend on the assumption that similar processes underlie both "same" and "different" responses. It may be, however, that these responses are based on different processes and/ or on different forms of information. A typical finding in studies requiring subjects to make "same" or "different" responses to pairs of stimuli is that "same" responses are faster than "different" responses (e.g., Bamber, 1969). This may be because different stimuli create inhibition between internal recognition or response processes (C. W. Eriksen, O'Hara, \& B. A. Eriksen, 1982; Proctor, 1981; Proctor \& Rao, 1983), or because subjects carry out a rechecking process before responding "different" (Krueger \& Shapiro, 1981). It is possible that either inhibition between the different shapes or the rechecking process overrules any effects due to the similarity of the shape orientations. For instance, although different shapes may be encoded most quickly when they are in the same orientation (because orientation information does not need to be recomputed and/or because they are more perceptually similar), the factors 
that facilitate encoding may slow down "different" decisions because of increased inhibition or longer rechecking times. The net result of these two antagonistic effects may be to mitigate any effects of orientation on "different" responses.

Analysis of the "different" response RTs failed to show any effect of the relative orientations of mismatching shapes. Also, "different" responses were consistently longer than "same" responses (see Table 1). These results accord with the notion that different processes were involved in "same" and "different" responses, and that the extra processes involved in "different" responses negate the effects of orientation found with "same" responses. Unfortunately, this claim rests upon acceptance of the null hypothesis for "different" responses. Inspection of the "different" response data indicates that sameorientation shapes were slightly harder to respond "different" to than were different-orientation shapes (Table 1), suggesting that there could have been a small bias towards "same" responses when shapes were in the same orientation. This possibility is examined more thoroughly in Experiments 3-5.

\section{EXPERIMENT 2}

One difficulty in accepting that the results of Experiment 1 reflect the effects of orientation on shape perception is that, with the short ISI used, apparent motion between the stimuli may have been present. It is possible that the effects were due to the properties of apparent motion, such as the relative spacing of the stimuli, etc. (Kolers, 1972); in this case, the argument that the effects of orientation change are pertinent to understanding shape perception is severely weakened. In order to investigate whether the results of Experiment 1 were due to differential apparent motion, Experiment 2 was conducted. A strategy similar to that adopted by Humphreys (1983) was used: the time course of apparent motion between shapes was contrasted with the time course of transformation effects on shape matching.

Transformation effects on shape matching were examined at three ISIs-50, 100, and $150 \mathrm{msec}$. In a separate session, the subjects rated the perceived apparent motion between the shapes at the different intervals. If the transformation effects on shape matching are determined by apparent motion, similar differences between the transformation conditions should emerge in both tasks, and the transformation effects should trace the same course over time. ${ }^{1}$

The use of three new ISIs in Experiment 2 also allowed the results of Experiment 1 to be generalized over a wider set of display timings.

\section{Method}

Six subjects, paid members of the Birkbeck College subject pool, participated. There were four males and two females, each with normal or corrected-to-normal vision. The subjects took part in two experimental sessions held on different days, with each session lasting about $40 \mathrm{~min}$.

The stimuli were the same as those used in Experiment 1 (Figure 2). In one experimental session, the subjects performed the same matching task as the subjects in the first study had. In the other session, subjects rated the quality of apparent motion between the consecutive shapes. Three ratings were possible, with each rating corresponding to a phenomenally distinct type of illusory movement. Response 1 signified rigid movement of the shape. This usually occurred between stimuli with the same shape. Response 2 signified nonrigid movement, often involving deformation of the contours in ST1 and ST2. This usually occurred between different shapes. Response 3 signified no apparent motion. This should have occurred with identical stimuli, which flickered but did not move. The subjects were instructed to use identical stimuli as the baseline for judging movement.

Ratings 1 and 2 were made by pressing the response buttons. When no response was made, rating 3 was recorded. The subjects were allowed an intertrial interval of $2.5 \mathrm{sec}$ to respond, which was easily sufficient for apparent movement to be rated. Following each response, the subjects were given feedback specifying RIGID MOVEMENT, NONRIGID MOVEMENT, or NO MOVEMENT, for $\mathbf{5 0 0 ~} \mathrm{msec}$ prior to the next display sequence, to reinforce the hand-response assignment.

For both sessions, there were three blocks of experimental trials, one at each ISI $(50,100$, and $150 \mathrm{msec})$, with 128 trials per block. Within each block, each stimulus was paired with every other stimulus and occurred first or second in the pair equally often, making 16 trials per condition per block. The stimulus and block of presentation orders were randomized for each subject. ST1 was presented for $50 \mathrm{msec}$ and ST2 for $200 \mathrm{msec}$. The hand-response assignment for both sessions was counterbalanced over subjects.

\section{Results}

The results for the two sessions were analyzed independently.

\section{Matching Task}

RT data: "Same" responses. The mean correct RTs for parallelograms and triangles, as a function of the transformation conditions and the ISI, are shown in Figure 3.

These data were analyzed in a three-way repeated measures design analysis of variance. Transformation condition (same position, vertical axis, horizontal axis, and same orientation), ISI $(50,100$, and $150 \mathrm{msec}$ ), and shape (parallelograms and triangles) were fixed factors; subjects were treated as a random factor. The main effect of transformation condition was statistically significant $[F(3,15)=7.67, p<.01]$. The main effects of ISI and shape were not reliable $[F(2,10)=2.88, p>.05$, and $F<1.0$, respectively], nor were any of the interactions.

Newman-Keuls analysis of the main effect of transformation condition showed that RTs in the sameposition and the same-orientation conditions were faster than those in the vertical-axis and the horizontalaxis conditions $(p<.05$ and $p<.01$, respectively). There were no differences between the same-position and same-orientation conditions, or between the vertical-axis and horizontal-axis conditions.

RT data: "Different" responses. The mean correct 

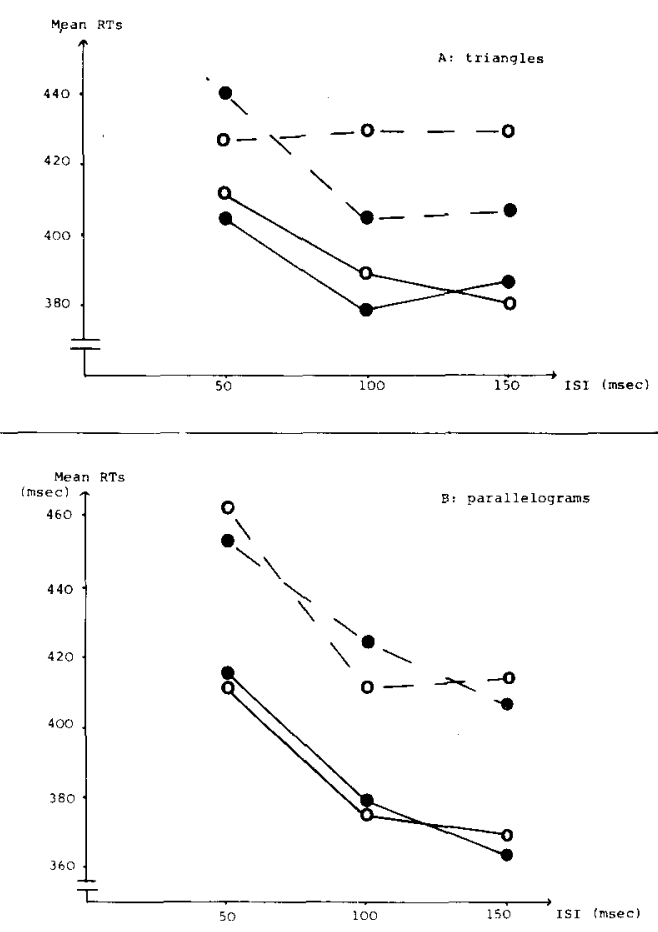

Figure 3. The mean correct RTs on "same" response trials in Experiment 2. A, triangles; B, parallelograms. Key: $-\bullet--$ tical-axis transformation; $\longrightarrow \mathrm{O} \longrightarrow$ same orientation.

RTs and percentage errors for different responses are given in Table 2.

There were no reliable main effects of transformation condition, ISI, or ST2 shape $[\mathrm{F}<1.0, \mathrm{~F}(2,10)=$ 2.85 , and $F(1,5)=1.50$, respectively; all ps $>.05$ ]. None of the interactions were reliable.

Error data. Only a small number of errors were made, and in general these followed the same trends as the RT data. For "same" responses, there were fewer errors in the same-position and same-orientation conditions $(1.2 \%$ and $1.9 \%$, respectively) than in the vertical-axis and horizontal-axis conditions $(2.3 \%$ and $2.5 \%$, respectively). There were no clear differences between the conditions for "different" responses.

\section{Apparent Motion}

Ratings of apparent motion were categorized as the proportion of trials on which each rating was made by each subject in each condition. When the stimuli were the same shape, the subjects typically used ratings 1 (rigid motion), when motion was seen, and 3 , when no motion was seen. When the stimuli differed in shape, the subjects typically used ratings 2 (nonrigid motion), when motion was seen, and 3, when no motion was seen.

The rating data were analyzed using analysis of variance techniques. This is because the $F$ test is relatively robust for violations of normality and homogeneity of variance in discrete frequency data, providing relatively large sample sizes are used (Bevan, Denton, \& Myers, 1974). Separate analyses were conducted for ratings between stimuli with the same shape (rigid motion) and stimuli with different shapes (nonrigid motion).

Rigid apparent motion. The proportion of trials on which rigid apparent motion was reported between parallelograms and between triangles is demonstrated in Figure 4.

A three-way repeated measures analysis of variance was performed, with ISI, transformation condition, and shape as fixed factors and subjects as a random factor. For this analysis, data from the sameposition condition were omitted because ratings of movement were made on only $.35 \%$ of the trials.

There were statistically significant main effects of ISI, transformation condition, and shape $[\mathrm{F}(2,16)=$ $15.64, p<.001 ; F(2,16)=9.04, p<.01 ;$ and $F(1,8)$ $=19.66, \mathrm{p}<.01$, respectively]. The interaction between ISI and the transformation conditions was also reliable $[F(4,32)=4.42, p<.01]$. None of the other interactions approached significance.

Newman-Keuls analysis of the main effect of ISI showed that more apparent motion was reported when the ISI was 50 or $100 \mathrm{msec}$ than when it was $150 \mathrm{msec}$ (both $\mathrm{p}<.01$ ). There was no reliable difference between the two shorter ISI conditions. A similar analysis of the main effect of transformation condition showed that more apparent motion was reported in the horizontal-axis condition than in the sameorientation and vertical-axis conditions (both ps $<.05$ ), which did not differ.

The ISI $x$ transformation condition was also examined using this technique. When the ISI was 50 msec, more movement was reported in the horizontalaxis condition than in the vertical-axis condition or

Table 2

Mean Correct RTs (in Milliseconds) and Percentage Errors (PE) on "Different" Response Trials as a Function of ST2 Shape, Transformation Condition, and ISI (in Milliseconds)

\begin{tabular}{|c|c|c|c|c|c|c|c|c|c|c|c|c|}
\hline \multirow[b]{3}{*}{ ST2 Shape } & \multicolumn{6}{|c|}{ Parallelogramis } & \multicolumn{6}{|c|}{ Triangles } \\
\hline & \multicolumn{2}{|c|}{50 ISI } & \multicolumn{2}{|c|}{100 ISI } & \multicolumn{2}{|c|}{150 ISI } & \multicolumn{2}{|c|}{50 ISI } & \multicolumn{2}{|c|}{100 ISI } & \multicolumn{2}{|c|}{150 ISI } \\
\hline & RT & PE & RT & PE & RT & PE & RT & PE & RT & $\mathrm{PE}$ & RT & PE \\
\hline Same Position & 511 & 0.7 & 457 & 1.2 & 464 & 1.0 & 483 & 1.2 & 439 & 0.7 & 423 & 1.1 \\
\hline Vertical-Axis Transformation & 481 & 1.8 & 438 & 1.5 & 471 & 1.6 & 467 & 1.9 & 445 & 1.8 & 424 & 1.7 \\
\hline Horizontal-Axis Transformation & 488 & 2.2 & 430 & 1.8 & 443 & 1.7 & 467 & 1.9 & 450 & 2.0 & 456 & 1.8 \\
\hline Same Orientation & 465 & 2.3 & 471 & 1.9 & 451 & 2.0 & 484 & 1.8 & 437 & 1.9 & 460 & 1.9 \\
\hline
\end{tabular}


the same-orientation condition $(p<.05$ and $p<.01$, respectively). There was no difference between the vertical-axis and the same-orientation conditions.

When the ISI was $100 \mathrm{msec}$, there was again more movement reported in the horizontal-axis condition than in the vertical-axis condition or the sameorientation condition (both ps $<.01$ ), which did not differ.

When the ISI was $150 \mathrm{msec}$, more movement was reported in the horizontal-axis condition than in the vertical-axis condition or the same-orientation condition ( $p<.01$ and $p<.05$, respectively). Also, more movement was now reported in the same-orientation condition than in the vertical-axis condition $(p<.05)$.

Nonrigid apparent motion. Similar analyses were performed on the ratings for nonrigid apparent motion. There were reliable main effects of ISI and transformation condition $[F(2,16)=12.39$ and $F(3.24)$ $=8.76$, ps $<.001$, respectively]. There was no effect of ST2 shape $(F<1.0)$. None of the interactions approached significance.

The main effects of ISI and transformation condition were further analyzed using the Newman-Keuls technique. Averaging over the shapes and transformation conditions, more apparent motion was reported at the short ISIs (50 and $100 \mathrm{msec}$ ) than at the longer ISI of $150 \mathrm{msec}(p<.01$ and $p<.05$, re-
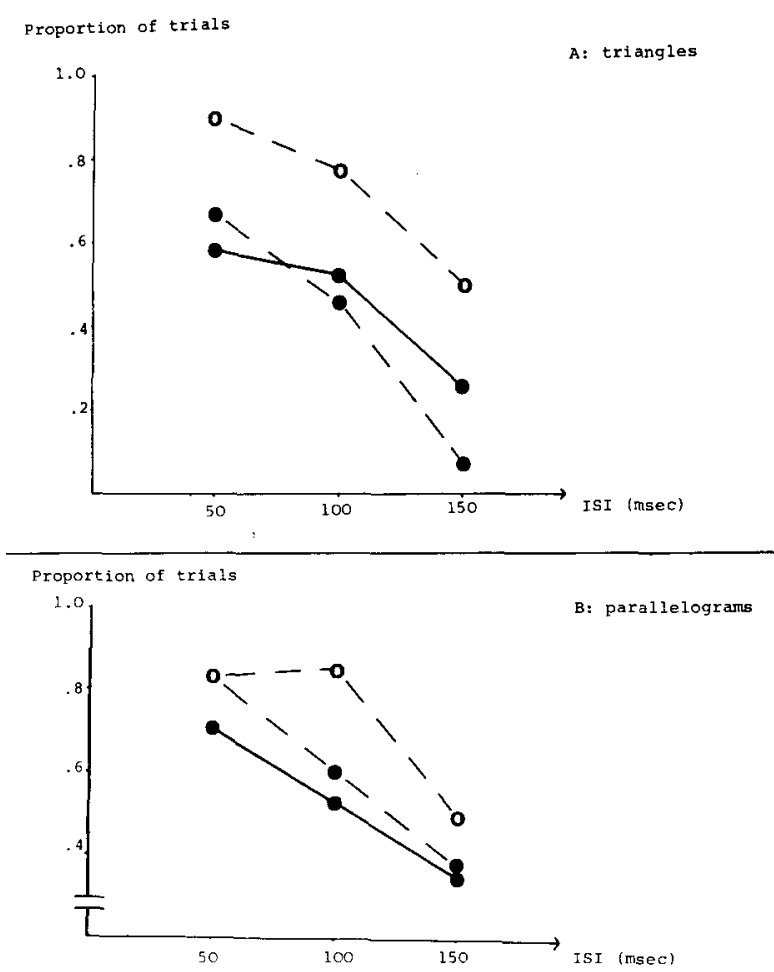

Figure 4. The proportion of trials on which apparent motion was reported between same-shape stimuli in Experiment 2. A, triangles; B, parallelograms. Key: - - 0 - - horizontal-axis transformation; - - - - vertical-axis transformation; - - same orientation.

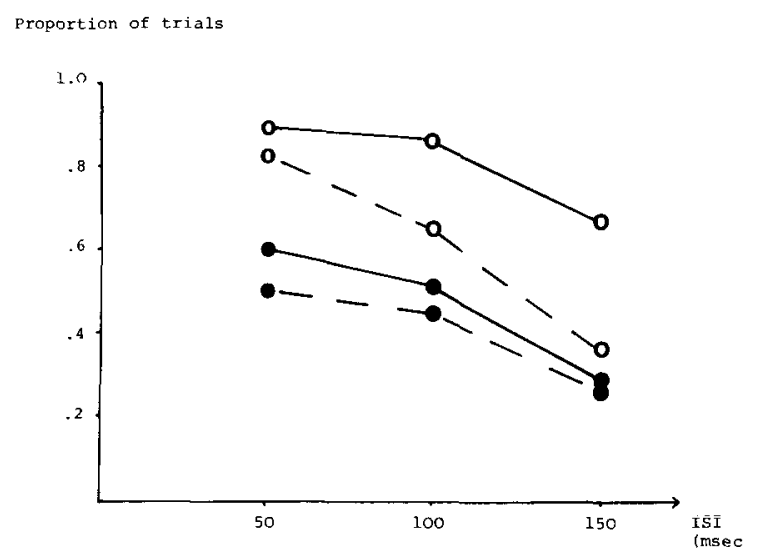

Figure 5. The proportion of trials on which apparent motion was reported between different-shape stimuli in Experiment 2. Key: - - O- same position; - - O - - horizontal-axis transformation; - - - - vertical-axis transformation; - - - same orientation.

spectively). The amount of apparent motion reported at the shorter intervals did not differ reliably. Analysis of the transformation effect showed that less apparent motion was reported in the vertical-axis and same-orientation conditions than in the same-position and horizontal-axis conditions ( $p<.01$ for comparisons with the same-position condition; $p<.05$ for comparisons with the horizontal-axis conditions). Apparent motion was also less in the horizontal-axis condition than in the same-position condition $(p<$ $.05)$. There were no other differences between the conditions.

Nonrigid apparent motion did not differ when ST2 was a parallelogram and when it was a triangle. Figure 5 illustrates the proportion of trials on which it was reported as a function of the transformation conditions and ISI, pooled over stimulus order.

\section{Discussion}

There are two important conclusions which can be drawn from Experiment 2. One is that the results of the matching task generalize over a range of ISIs from 50 to $200 \mathrm{msec}$. As in Experiment 1, RTs in the sameposition and the same-orientation conditions were faster than those in the vertical-axis and the horizontalaxis conditions. This difference was constant over the ISIs studied, demonstrating the robust effects of orientation change on matching performance. Compared with the effects of orientation change, changing the position of the focal feature from the top to the bottom of the shape had little disruptive effect. The trend for faster matching in the vertical-axis condition than in the horizontal-axis condition was present but nonsignificant. More importantly, there were again no differential transformation effects on "different" responses. There is also no indication in the data that "different" responses were more 
difficult to make for same-orientation shapes than for shapes in different orientations (Table 2). This is consistent with the assertion that "different" and "same" responses reflect different processes, and that the processes involved in responding "different" to two shapes overrule any effect of their having the same orientation.

The second important finding is that transformation type and ISI produced different effects on perceived apparent motion and on shape matching. Rigid apparent motion was seen only between matching shapes. Irrespective of the ISI, rigid motion was seen most often in the horizontal-axis condition, and it did not differ for the same-orientation and verticalaxis conditions except at the long (150-msec) ISI. Nonrigid motion was seen between different shapes. It was seen most between shapes in the same-position condition, less between shapes in the horizontal-axis condition, and least in the vertical-axis and sameorientation conditions. Both forms of apparent motion decreased as the ISI lengthened.

The divergence between apparent motion ratings and shape-matching performance illustrates that independent mechanisms underlie these tasks. Apparent motion appears to have been affected primarily by the relative distance between ST1 and ST2. Thus, the strongest rigid motion was seen between shapes in the horizontal-axis condition, because those shapes were closer than were the shapes in the other transformation conditions. The strongest nonrigid motion was seen in the same-position condition, in which ST1 and ST2 were even closer than in the horizontalaxis condition. On the other hand, shape matching was affected by whether the shapes were presented in the same orientation. It seems reasonable to conclude from this that the orientation effects are specific to the processes involved in shape perception and matching.

\section{EXPERIMENT 3}

Although the results of Experiment 2 suggest that orientation effects are a product of shape perception and matching, they are not informative about the particular mechanisms involved. For instance, one possibility is that the data reflect obligatory aspects of shape perception (such as those involved in the construction and maintenance of axis-based descriptions). Another possibility, though, is that the data reflect an arbitrary strategy adopted by subjects to discriminate matching and mismatching shapes. The data from Experiments 1 and 2 indicate that any effects were not due to the use of a lower "same" response criterion for shapes in the same orientation, since there were no transformation effects on "different" responses. However, this argument depends on the acceptance of the null hypothesis. A more conclusive test of strategic effects on matching would be to present the transformation conditions in differ- ent trial blocks. In this case, subjects should adopt an optimal strategy for each condition and any differences between the conditions due to the type of strategy adopted should decrease. This was the purpose of Experiment 3, in which the transformation conditions were run in separate blocks.

\section{Method}

Twelve paid members of the Birkbeck College subject pool participated in the experiment. There were seven males and three females, and each had normal or corrected-to-normal vision. The subjects took part in a single experimental session lasting about $40 \mathrm{~min}$.

The stimuli were the same as in Experiments 1 and 2 (Figure 2). However, in contrast to those experiments, the three transformation conditions were presented in separate blocks of trials. For each subject, the experimental session consisted of three blocks of 192 experimental trials. Within each block, stimuli were presented in the same position on half the trials and in a position dictated by the transformation condition on the other half. In each block, there were 96 trials on which the stimuli had the same number of sides; there were 48 "same" response trials for parallelograms and for triangles, 24 in the same-position condition and 24 in the transformation condition (vertical-axis, horizontal-axis, and same orientation). Within each condition, each possible stimulus occurred equally often as ST1 and ST2. The same-position condition served as the baseline from which to measure the relative effect of the other transformation conditions in each block. The order of the blocks was randomized for each subject. Prior to the experimental trials in each block, the subjects received 32 practice trials in the appropriate transformation condition.

The stimulus durations used in Experiment 1 were employed. The subjects were instructed to match consecutive shapes on the basis of the number of sides they contained. Half the subjects responded "same" with the preferred hand, half responded "different."

\section{Results}

\section{RT Data: “Same"' Responses}

The mean correct RTs and percentage errors in each block of trials for matching shapes are given in Table 3. RTs in each block are broken down into those made to same-position stimuli and those made to stimuli in the transformation condition.

RTs were analyzed in a three-way repeated measures analysis of variance. Trial block (horizontalaxis, vertical-axis, same orientation), shape (parallelogram and triangle), and condition (same position and transformed) were fixed effects, and subjects were treated as a random factor. The main effects of trial block and shape were not statistically significant $[F(2,22)=1.09$ and $F(1,11)=2.45$, respectively; both ps $>.05]$. The main effect of condition was reliable $[F(1,11)=5.90, p<.05]$, demonstrating that $R T s$ in each transformation condition were slower than RTs to shapes in the same position. Importantly, none of the interactions were statistically significant.

\section{RT Data: "Different"' Responses}

The mean correct RTs and percentage errors for mismatching shapes are presented in Table 3.

There were no reliable effects of trial block, shape, 
Table 3

Mean Correct RTs (in Milliseconds) and Percentage Errors (PE) on "Same" and "Different" Response Trials in Each Block of Trials in Experiment 3

\begin{tabular}{|c|c|c|c|c|c|c|c|c|c|c|c|c|}
\hline & \multicolumn{12}{|c|}{ Trial Block } \\
\hline & \multicolumn{4}{|c|}{ Horizontal-Axis Transformation } & \multicolumn{4}{|c|}{ Vertical-Axis Transformation } & \multicolumn{4}{|c|}{ Same-Orientation Transformation } \\
\hline & \multicolumn{2}{|c|}{ Same Position } & \multicolumn{2}{|c|}{ Transformed } & \multicolumn{2}{|c|}{ Same Position } & \multicolumn{2}{|c|}{ Transformed } & \multicolumn{2}{|c|}{ Same Position } & \multicolumn{2}{|c|}{ Transformed } \\
\hline & RT & PE & RT & PE & RT & $\mathrm{PE}$ & RT & $\mathrm{PE}$ & RT & PE & RT & PE \\
\hline \multicolumn{13}{|c|}{ "Same" Response Trials } \\
\hline $\begin{array}{l}\text { Parallelogram } \\
\text { Triangle }\end{array}$ & $\begin{array}{l}503 \\
490\end{array}$ & $\begin{array}{l}2.2 \\
1.9\end{array}$ & $\begin{array}{l}523 \\
508\end{array}$ & $\begin{array}{l}2.4 \\
2.3\end{array}$ & $\begin{array}{l}544 \\
494\end{array}$ & $\begin{array}{l}1.9 \\
2.3\end{array}$ & $\begin{array}{l}557 \\
518\end{array}$ & $\begin{array}{l}2.1 \\
2.6\end{array}$ & $\begin{array}{l}521 \\
506\end{array}$ & $\begin{array}{l}1.9 \\
2.1\end{array}$ & $\begin{array}{l}541 \\
531\end{array}$ & $\begin{array}{l}1.8 \\
2.2\end{array}$ \\
\hline \multicolumn{13}{|c|}{ "Different" Response Trials } \\
\hline $\begin{array}{l}\text { Parallelogram } \\
\text { Triangle }\end{array}$ & $\begin{array}{l}538 \\
530\end{array}$ & $\begin{array}{l}0.7 \\
0.8\end{array}$ & $\begin{array}{l}536 \\
539\end{array}$ & $\begin{array}{l}0.9 \\
1.0\end{array}$ & $\begin{array}{l}560 \\
553\end{array}$ & $\begin{array}{l}0.8 \\
1.1\end{array}$ & $\begin{array}{l}559 \\
558\end{array}$ & $\begin{array}{l}1.0 \\
1.3\end{array}$ & $\begin{array}{l}567 \\
566\end{array}$ & $\begin{array}{l}1.0 \\
0.9\end{array}$ & $\begin{array}{l}552 \\
558\end{array}$ & $\begin{array}{l}0.9 \\
1.1\end{array}$ \\
\hline
\end{tabular}

or condition $[F(2,22)=1.45, F<1.0$, and $F(1,11)=$ 1.02 , respectively; all ps $>.05]$. None of the interactions approached statistical significance.

\section{Error Data}

The errors matched the RT results. For example, for "same" responses, more errors were made when the shapes were transformed than when they were in the same position. There were few differences between the transformation conditions (Table 3). Because of the small numbers involved and the similarity between these and the RT data, no further analyses were performed.

\section{Discussion}

Contrary to the outcomes in Experiments 1 and 2, there were no differences between the transformation conditions in this study; also, RTs in all the transformation conditions were slower than RTs in the same-position condition. These results suggest that the earlier findings were dependent on the particular stimulus set used, perhaps indicating the nature of the similarity space adopted by subjects (Lockhead, 1972). When the transformation conditions are presented to subjects at random, as in Experiments 1 and 2, RTs may be affected by similarity relations between all the stimuli in the set. These relations appear to favor stimuli presented in the same orientation. The effect of blocking the transformations, as in Experiment 3, would be to confine the similarity space to same-position stimuli and to the stimuli in the particular transformation condition. This may be expected to distance same-position stimuli from transformed stimuli and to minimize differences between the transformation effects. While it is of interest to understand why same-orientation stimuli might be grouped within a similarity space under some circumstances, this argument does suggest that the results do not reflect obligatory processes in shape perception, because the nature of a particular similarity space would be specific to the experiment.
However, alternative arguments can be proposed to account for the data. For instance, it may be that blocking the transformation conditions allowed subjects to attend selectively to distinguishing local characteristics of the shapes and to ignore global shape characteristics such as orientation. This possibility exists because blocking the conditions in Experiment 3 reduced uncertainty about the spatial locations of the stimuli, since ST2 could occur in only one of two quadrants. With the display durations used, subjects may have re-allocated attention following ST1 to the informative local features in ST2.

One way to separate these accounts is to increase uncertainty about the spatial locations of stimuli while maintaining blocked presentation of the transformation conditions. If the failure to find differential transformation effects in Experiment 3 was because subjects could selectively attend to focal feature locations, increasing the locational uncertainty should restore the effects.

\section{EXPERIMENT 4}

In Experiment 4, transformation conditions were blocked, as in Experiment 3, but there was a greater degree of uncertainty about the spatial location of possible targets. ST1 was always centered at fixation. To prevent selective attention to the expected location of a focal feature, ST2 was presented randomly at one of four locations equidistant from fixation. To prevent the use in Experiment 4 of particular strategies subjects might have used to match shapes in Experiments 1-3, ST2 was presented at a location some distance from ST1. For instance, subjects could not then base decisions on the equality of angles meeting at fixation, or the presence of a matching diagonal through fixation, both of which strategies could have selectively benefited the same-orientation condition.

\section{Method}

There were 12 subjects, seven female and five male, all paid members of the Birkbeck College subject pool. All subjects had 
either normal or corrected-to-normal vision, and each took part in a single experimental session lasting about $\mathbf{4 0} \mathrm{min}$.

The stimuli were the same as those used previously. However, ST1 was always centered at fixation and ST2 at a location $\pm 10 \mathrm{~cm}$ away on both $\mathrm{X}$ and $\mathrm{Y}$ coordinates in one of the four quadrants selected at random.

As in Experiment 3, there was blocked presentation of the vertical-axis, horizontal-axis, and same-orientation transformation conditions. There were three blocks of 128 experimental trials. Within each block, stimuli were in the same position on half the trials and were transformed on the other trials; on half the sameposition and transformation trials, they were the same shape, and on the other half, they were different shapes. Within each condition, each stimulus was equally often ST1 and ST2, and, when ST2, each was presented equally often at each location. Prior to each block, the subjects were given 32 practice trials in the particular transformation condition, and the order of blocks was randomized over subjects.

Stimulus durations were the same as in Experiments 1 and 3. Half the subjects responded "same" with the preferred hand; the response-hand assignment was reversed for the other subjects.

\section{Results}

\section{RT Data: “'Same"' Responses}

Responses for each shape in each condition in each trial block were averaged over the four location positions for ST2. The mean correct RTs and percentage errors are presented in Table 4.

There was a reliable difference between performance in the horizontal-axis, vertical-axis, and sameorientation trial blocks $[\mathrm{F}(2,22)=4.25, \mathrm{p}<.05]$; there was also a reliable difference between the sameposition and the transformation conditions $[F(1,11)$ $=25.22, \mathrm{p}<.001]$. The only other reliable effect was the interaction between the trial block and the transformation/same-position difference $[F(2,22)=7.66$, $\mathrm{p}<.01]$.

Pairwise comparisons were conducted to analyze the transformation/same-position differences as a function of the trial block. There was a reliable difference between the size of the transformation effect in the same-orientation block and the vertical-axis block $[F(1,22)=6.46, p<.025]$ and in the sameorientation block and the horizontal-axis block $[\mathrm{F}(1,22)$
$=5.40, \mathrm{p}<.05]$, but not in the horizontal-axis and vertical-axis blocks $(\mathrm{F}<1.0)$.

\section{RT Data: "Different"' Responses}

The mean correct RTs and percentage errors are given in Table 4.

There were no reliable effects of trial block, transformation effect, or ST2 shape $[F<1.0, F(1,11)=$ 2.62 , and $F(1,11)=2.27$, respectively; all ps $>.05$ ] None of the interactions were statistically significant.

\section{Error Data}

As in all the present experiments, low error rates were recorded. There was a tendency for more errors to occur in the transformation conditions than in the same-position conditions (Table 4), but there were no other marked differences between the conditions. There was no indication of a speed-error tradeoff.

\section{Discussion}

The data from this experiment demonstrate that blocking the transformation conditions does not eliminate the differential transformation effects, providing subjects remain uncertain about the spatial location of ST2. The data replicate the finding obtained in Experiments 1 and 2 that same-orientation transformations were less detrimental to shape matching than were vertical-axis and horizontal-axis transformations. Also, as in the first two experiments, performance in the same-orientation condition was similar to that in the same-position condition. This indicates that the failure to find these results in Experiment 3 was because subjects could selectively attend to the location of focal features in ST2, and not because the transformation conditions were blocked. The fact that orientation change affected performance even with a reduced stimulus set suggests that the effect is not due primarily to the interstimulus similarities present in the particular experiment.

The contrast between Experiments 3 and 4 demonstrates interesting limits on the conditions under

Table 4

Mean Correct RTs (in Milliseconds) and Percentage Errors (PE) on "Same" and "Different" Response Trials in Each Block of Trials in Experiment 4

\begin{tabular}{|c|c|c|c|c|c|c|c|c|c|c|c|c|}
\hline & \multicolumn{12}{|c|}{ Trial Block } \\
\hline & \multicolumn{4}{|c|}{ Horizontal-Axis Transformation } & \multicolumn{4}{|c|}{ Vertical-Axis Transformation } & \multicolumn{4}{|c|}{ Same-Orientation Transformation } \\
\hline & \multicolumn{2}{|c|}{ Same Position } & \multicolumn{2}{|c|}{ Transformed } & \multicolumn{2}{|c|}{ Same Position } & \multicolumn{2}{|c|}{ Transformed } & \multicolumn{2}{|c|}{ Same Position } & \multicolumn{2}{|c|}{ Transformed } \\
\hline & RT & PE & RT & $\mathrm{PE}$ & $\mathrm{RT}$ & $\mathrm{PE}$ & RT & PE & RT & $\mathrm{PE}$ & RT & $\mathrm{PE}$ \\
\hline \multicolumn{13}{|c|}{ "Same" Response Trials } \\
\hline Parallelogram & 438 & 2.2 & 452 & 2.6 & 412 & 2.3 & 456 & 2.5 & 413 & 2.1 & 421 & 2.2 \\
\hline Triangle & 439 & 1.9 & 495 & 1.8 & 415 & 2.1 & 450 & 2.4 & 414 & 2.0 & 415 & 2.1 \\
\hline \multicolumn{13}{|c|}{ “Different" Response Trials } \\
\hline Parallelogram & 512 & 1.9 & 537 & 2.2 & 512 & 2.1 & 510 & 2.3 & 506 & 2.0 & 499 & 2.2 \\
\hline Triangle & 542 & 1.6 & 514 & 2.4 & 509 & 2.1 & 497 & 2.8 & 549 & 1.8 & 514 & 2.3 \\
\hline
\end{tabular}


which orientation effects influence shape matching. Orientation effects occur when there is spatial uncertainty and subjects cannot selectively attend to the focal features in ST2; orientation effects can be eliminated when subjects can attend to the locations of the focal features discriminating the to-be-matched shapes. One account of this contrast is that subjects use global structural characteristics, such as the orientation of the principal axis, for shape representation in conditions of high spatial uncertainty; they use local structural characteristics, such as the identity and location of a focal feature, in conditions of low spatial uncertainty.

\section{EXPERIMENT 5}

Experiments 1-4 were concerned with establishing the conditions under which orientation change affects shape matching using two shapes, triangles and parallelograms. It is important to demonstrate that the results also hold for other shapes with a principal elongated axis, to show that they illustrate processes general to the perception and matching of elongated shapes. Experiment 5 was conducted for this purpose. Two new shapes were used (Figure 6). Both shapes were generated from a rectangular base; in one case one right-angled triangle was added to the base, and in the other two right-angled triangles were
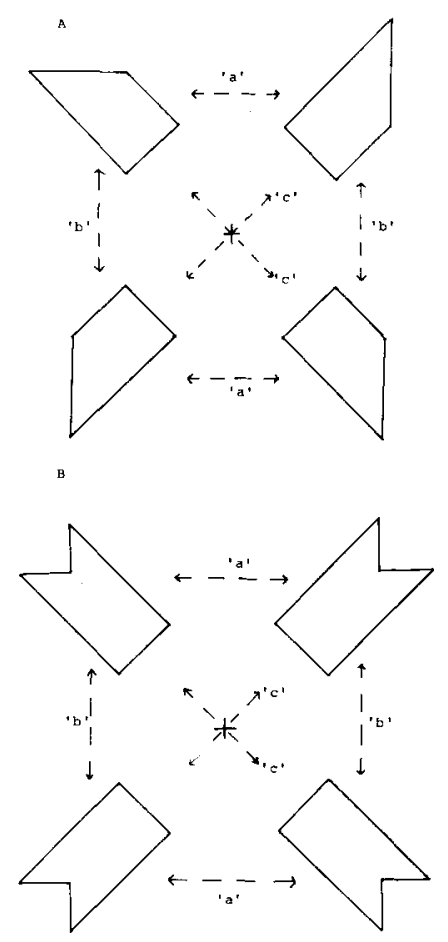

Figure 6. The shapes and transformation conditions used in Experiment 5. Transformation $a=$ vertical-axis condition; transformation $b=$ horizontal-axis condition; transformation $c=$ sameorientation condition. added. This created two shapes, each with a principal elongated axis, differing in one focal feature (the presence of the apex of the second triangle). These shapes were subjected to transformations similar to those used in the previous studies; however, the transformations operated about four points equidistant from fixation. The shapes used in Experiment 5 had two particular attributes that made them suitable for the study: they could not be matched on the basis of focal features near fixation, and they allowed a direct test of the effects of left-right direction change on matching. Single apex shapes in the vertical-axis condition ( $a$ in Figure 6A) changed their left-right direction relative to their focal feature on only half the trials (bottom right $\leftrightarrow$ bottom left quadrant). Similarly, single apex shapes in the horizontal-axis condition (b in Figure 6A) changed their left-right direction on half the trials (top right $\rightarrow$ bottom right). If left-right direction change affects matching, RTs should be slowest on the direction-change trials.

\section{Method}

There were 12 subjects, seven male and five female, all paid members of the Birkbeck College subject pool. All subjects had normal or corrected-to-normal vision and took part in one experimental session of about 35 min duration.

The subjects were presented with shapes from the set shown in Figure 6. These shapes were created in the same manner as the earlier shapes, with the exception of the shape in the bottom lefthand quadrant, which was produced by a 180-deg rotation of the top-right-quadrant shape about the y-axis followed by a 90 -deg rotation within the plane, about a point $10 \mathrm{~cm}$ to the left and below fixation. This created left-right direction changes for all the trials in the same-orientation condition and for half the trials in the horizontal-axis and vertical-axis conditions.

The transformation conditions were presented at random. There were three blocks of experimental trials, with 128 trials per block (384 trials). Within each block, each stimulus was paired with every other stimulus equally often and was presented first and second in that pairing twice. Subjects were told the set of stimuli, and were instructed to respond "same" if the two shapes on a trial were physically identical irrespective of their relative orientations. Half the subjects used the preferred hand to respond "same," and half used the preferred hand to respond "different." ST1 was presented for $50 \mathrm{msec}$ and ST2 for $200 \mathrm{msec}$, and there was a blank ISI of $200 \mathrm{msec}$.

\section{Results}

\section{RT Data: "Same" Responses}

In Table 5, the mean correct RTs as a function of the transformation condition and shape are presented. A two-way repeated measures analysis of variance was carried out. Condition (same position, vertical-axis transformation, horizontal-axis transformation, and same orientation) and shape (single and dual apex) were fixed effects, and subjects were treated as a random factor. The main effect of condition was statistically significant $[F(3,33)=4.60$, $p<.01]$. The effect of shape was not significant, and nor was the shape $\times$ condition interaction $[F(1,11)$ $=2.82$ and $F(3,33)=2.74$, respectively; both ps $>$.05]. 
Newman-Keuls analysis of the condition effect showed that RTs were faster in the same-position and the same-orientation conditions than in the horizontalaxis and vertical-axis conditions $(p<.01$ for the same-orientation and horizontal-axis comparison, $\mathrm{p}<.05$ for all other comparisons). Same-position and same-orientation conditions and vertical-axis and horizontal-axis conditions did not differ.

To investigate the effects of left-right direction change on performance, RTs in the horizontal-axis and vertical-axis conditions for the single apex shape were separated according to whether there was a leftdirection change. No effects were apparent. In the vertical-axis condition, the mean RT on same-direction trials was $535 \mathrm{msec}$, and on different-direction trials, it was $544 \mathrm{msec}$. In the horizontal-axis condition, the mean RT on same-direction trials was $548 \mathrm{msec}$, and on different-direction trials, it was $545 \mathrm{msec}$. These differences did not approach significance (both $\mathrm{F}<1.0$ ).

\section{RT Data: “Different" Responses}

Similar analyses were performed on the "different" response RTs. Neither the main effects of condition or ST2 nor the condition $\times$ shape interaction were reliable $[F(3,33)=2.81, F(1,11)=2.24$, and $F(3,33)=$ 2.48 , respectively; all ps $>.05$ ]. The mean correct RTs as a function of the condition and ST2 shape are given in Table 5 .

\section{Error Data}

The error rate in this study was slightly higher than in the previous experiments. This was most likely because the discrimination between the shapes here was more difficult than the discrimination between parallelograms and triangles. Nevertheless, error rates followed the same trends as the RT data (Table 5). RTs were not traded off against response accuracy.

\section{Discussion}

The data replicate the findings of Experiments 1,
2 , and 4 with new stimuli. RTs were faster to the same-shape stimuli in the same-position and the sameorientation conditions than to the same-shape stimuli when they were transformed about either the horizontal or the vertical axis.. Also, RTs did not differ in the same-position and the same-orientation conditions. This demonstrates that the results are not confined to the particular stimulus set and discrimination task used.

Experiment 5 also confirms that, in the present task, changes in the left-right direction of parts of shapes have little effect on performance. One possible reason for this is that we may not normally have an explicit representation of the left-right direction of a shape (Hinton \& Parsons, 1981). Such a representation may be useful for a more limited number of tasks (such as discriminating shapes from their reflections), and its use may require operations quite different from those investigated here (such as mental rotation).

\section{GENERAL DISCUSSION}

Four experiments (Experiments 1, 2, 4, and 5) have been reported showing that the time taken to match consecutive orientation-transformed shapes is less when the orientations of their principal axes are constant than when they change. When this effect occurs, performance is not affected by the absolute locations of the shapes in the visual field: when the shapes' orientations are constant but their positions change, RTs are equivalent to those obtained when their positions are the same. Orientation effects were not apparent with "different" responses. This suggests that they were not caused by a bias toward responding "same" to shapes in the same orientation. Also, the effects were not related to differences in apparent motion between the stimuli (Experiment 2), and they are unlikely to be due to similarity relations between stimuli in the particular experiment (Experiment 4).

Taken together, the results suggest that presenting matching shapes in the same orientation increases their perceptual similarity relative to when they are

Table 5

Mean RTs (in Milliseconds) and Percentage Errors (PE) for "Same" and "Different" Response Trials in Experiment 5

\begin{tabular}{|c|c|c|c|c|}
\hline & \multicolumn{2}{|c|}{ Parallelogram } & \multicolumn{2}{|c|}{ Triangle } \\
\hline & RT & $\mathrm{PE}$ & RT & PE \\
\hline \multicolumn{5}{|c|}{ “Same” Response Trials } \\
\hline $\begin{array}{l}\text { Same Position } \\
\text { Vertical-Axis Transformation } \\
\text { Horizontal-Axis Transformation } \\
\text { Same Orientation }\end{array}$ & $\begin{array}{l}513 \\
540 \\
546 \\
512\end{array}$ & $\begin{array}{l}2.6 \\
3.5 \\
3.4 \\
2.9\end{array}$ & $\begin{array}{l}539 \\
563 \\
581 \\
525\end{array}$ & $\begin{array}{l}2.8 \\
3.9 \\
4.1 \\
2.8\end{array}$ \\
\hline \multicolumn{5}{|c|}{ "Different" Response Trials } \\
\hline $\begin{array}{l}\text { Same Position } \\
\text { Vertical-Axis Transformation } \\
\text { Horizontal-Axis Transformation } \\
\text { Same Orientation }\end{array}$ & $\begin{array}{l}622 \\
574 \\
570 \\
572\end{array}$ & $\begin{array}{l}3.2 \\
3.4 \\
2.9 \\
3.1\end{array}$ & $\begin{array}{l}570 \\
564 \\
557 \\
576\end{array}$ & $\begin{array}{l}3.6 \\
2.9 \\
3.3 \\
3.2\end{array}$ \\
\hline
\end{tabular}


presented in different orientations. One reason for this might be that subjects generate axis-based shape descriptions and maintain information about the orientation of the axis. RTs to match shapes may then be facilitated by presentation of the shape in the same orientation because orientation information does not have to be recomputed. The simplest explanation for the failure to find orientation effects on "different" responses, then, is that "different" responses involve more processes than "same" responses. Typically, "different" responses were slower. It may be that presenting different shapes in the same orientation facilitates the computation of their shape descriptions because orientation information does not have to be respecified. However, matching orientation information may also activate internal recognition or response processes that are incompatible with those activated by the mismatching shape descriptions (C. W. Eriksen et al., 1982; Proctor, 1981). Inhibition at the decision stage may cancel out any facilitation of earlier processes.

The orientation effects on "same" responses are specific to shape matching and perception, since quite different effects were found with apparent motion ratings. Apparent motion appeared to be determined by the spatial proximity of the stimuli, with more motion occurring for stimuli in close proximity, both when same and when different shapes were presented. This suggests that apparent motion was due to processes concerned with local disparity detection and not the global structural properties of the shapes (e.g., Braddick, 1974). From this it seems that independent mechanisms can underlie shape perception and apparent motion.

Interestingly, orientation effects do not generalize to all instances of shape perception. The effects occur primarily when subjects are uncertain of the spatial location of the to-be-matched shapes (Experiments 1, 2,4 , and 5). Under this circumstance, subjects appear to use the global property of principal-axis orientation to represent shapes internally. When spatial uncertainty is reduced, though, subjects appear to base shape representations upon salient local features, minimizing orientation effects (Experiment 3). This result is important because it suggests that spatial attention can play a functional role in shape perception.

The present results were obtained using tasks in which shape direction (up-down, left-right) was not relevant for response purposes. However, this will not be the case for all tasks or all stimuli. Clearly, shape direction must be represented when subjects have to discriminate between reflected stimuli; it may also be important for simple physical matching tasks with particular stimuli (such as faces; Yin, 1969). One necessary question for future research, then, is whether the present effects change according to the nature of the stimulus. This suggests the interesting possibility that object perception and matching can be influenced by the functional characteristics of the object.

\section{REFERENCES}

Bamber, D. (1969). Reaction times and error rates for "samedifferent" judgments of multidimensional stimuli. Perception \& Psychophysics, 6, 169-174.

Bеск, J. (1972). Similarity grouping and peripheral discriminability under uncertainty. American Journal of Psychology, 85, 1-20.

Bevan, M. F., Denton, J. Q., \& Myers, J. L. (1974). The robustness of the $F$ test to violations of continuity and form of treatment population. British Journal of Mathematical and Statistical Psychology, 27, 199-204.

Braddick, O. (1974). A short-range process in apparent motion. Vision Research, 14, 519-527.

Braine, L. G. (1978). A new slant on opientation perception. American Psychologist, 33, 10-22.

Braine, L. G., Relyea, L., \& Davidman, L. (1981). On how adults identify the orientation of a shape. Perception \& Psychophysics, 29, 138-144.

Bundesen, C., \& Larsen, A. (1975). Visual transformation of size. Journal of Experimental Psychology: Human Perception and Performance, 1, 214-220.

Cooper, L. A., \& Shepard, R. N. (1973). Chronometric studies of the rotation of mental images. In W. G. Chase (Ed.), Visual information processing. New York: Academic Press.

Cooper, L. A., \& ShePARd, R. N. (1975). Mental transformations in the identification of left and right hands. Journal of Experimental Psychology: Human Perception and Performance, 1, 221-230.

Eriksen, C. W., O'Hara, W. P., \& Eriksen, B. A. (1982). Response competition effects in same-different judgments. Perception \& Psychophysics, 32, 261-270.

Goldmeier, E. (1936). Uber Ahnlichkeit be: gesehenen Figuren. Psychologische Forschung, 21, 146-208.

Hinton, G. E. (1981). A parallel computation that assigns canonical object-based frames of reference. In Proceedings of the Seventh International Joint Conference on Artificial Intelligence (Vancouver, B.C., Canada).

Hinton, G. E., \& Parsons, L. M. (1981). Frames of reference and mental imagery. In J. Long \& A. Baddeley (Eds.), Attention and performance $I X$. Hillsdale, $\mathrm{NJ}$ : Erlbaum.

Humphreys, G. W. (1983). Reference frames and shape perception. Cognitive Psychology, 15, 151-196.

Julesz, B. (1971). Foundations of cyclopean perception. Chicago: University of Chicago Press.

Julesz, B., Gilbert, E. N., Shepp, L. A., \& Frisch, H. L. (1973). Inability of humans to discriminate between visual textures that agree in second-order statistics-revisited. Perception, 2, 391-405.

Kole rs, P. A. (1972). Aspects of motion perception. New York: Pergamon.

Krueger, L. (1978). A theory of perceptual matching. Psychological Review, 85, 278-304.

Krueger, L. E., \& Shapiro, R. G. (1981). A reformulation of Proctor's unified theory for matching-task phenomena. Psychological Review, 88, 573-581.

Latsen, A., \& Bundesen, C. (1978). Size scaling in visual pattern recognition. Journal of Experimental Psychology: Human Perception and Performance, 4, 1-20.

Locknead, G. R. (1972). Processing dimensional stimuli: A note. Psychological Review, 79, 410-419.

МАCH, E. (1897). Contributions to the analysis of sensations. Chicago: Open Court. 
MARR, D. (1979). Representing and computing visual information. In P. H. Winston \& R. H. Brown (Eds.), Artificial intelligence: An MIT perspective (Vol. 2). London: MIT Press.

MARR, D. (1980). Visual information processing: The structure and creation of visual representations. Philosophical Transactions of the Royal Society (London), B, 290, 199-218.

MARR, D. (1982). Vision. San Francisco: Freeman.

MARR, D., \& NishiharA, H. K. (1978). Representation and recognition of the spatial organisation of three-dimensional shapes. Proceedings of the Royal Society (London), B, 200, 269-294.

Metzler, J., \& Shepard, R. N. (1974). Transformational studies of the internal representation of three-dimensional objects. In $\mathrm{R}$. Solso (Ed.), Theories in cognitive psychology: The Loyola symposium. Potomac, MD: Erlbaum.

Proctor, R. W. (1981). A unified theory for matching-task phenomena. Psychological Review, 88, 291-326.

Procton, R. W., \& RAO, K. V. (1983). Null effects of exposure duration and heterogeneity of difference on the same-different disparity in letter matching. Perception \& Psychophysics, 33, 163-171.

Rock, I., DiVita, J., \& Barbeito, R. (1981). The effect on form perception of change of orientation in the third dimension. Journal of Experimental Psychology: Human Perception and Performance, 7, 719-732.

Rock, I., \& Leaman, R. (1963). An experimental analysis of visual symmetry. Acta Psychologia, 21, 171-183.
Seruler, R., \& Nash, D. (1972). Speed of size scaling in human vision. Psychonomic Science, 27, 93-94.

SHEPARD, R. N. (1975). Form, formation, and transformation of internal representations. In R. Solso (Ed.), Information processing and cognition: The Loyola symposium. Hillsdale: $\mathrm{NJ}$ Erlbaum.

She Pard, R. N., \& Metzler, J. (1971). Mental rotation of threedimensional objects. Science, 171, 701-703.

YIN, R. K. (1969). Looking at upside down faces. Journal of EXperimental Psychology, 81, 141-145.

\section{NOTE}

1. Another possible strategy to assess the effects of apparent motion on shape matching, suggested by one referee, would be to use ISIs sufficiently long to prohibit apparent motion. Unfortunately, this procedure would then be more likely to demand memorial representations than perceptual ones of stimuli. Also, with longer ISIs, subjects may perform internal transformations upon ST1 to match it with ST2 (Humphreys, 1983). RTs in the matching task would then be less likely to reflect the similarity of the perceptual representations determined by the stimuli.

(Manuscript received July 1983; revision accepted for publication May 30, 1984.) 